\title{
INFLUENCIA DE LA PERCEPCIÓN Y METODOLOGÍAS DOCENTES APLICADAS EN EL RENDIMIENTO ACADÉMICO DE LOS ESTUDIANTES DE LA ASIGNATURA DE DIRECCIÓN DE RECURSOS HUMANOS EN LAS EMPRESAS TURÍSTICAS
}

\author{
María de los Ángeles Frende Vega* \\ José María Biedma Ferrer** \\ Manuel Arana Jiménez**** \\ Universidad de Cádiz
}

\section{RESUMEN}

En este trabajo se pretende estudiar los factores que influyen en el rendimiento académico de los estudiantes de la asignatura de Dirección de Recursos Humanos en Empresas Turísticas del Grado de Turismo de la Universidad de Cádiz. En concreto, analizamos si la percepción que tienen sobre la materia tiene relación con el esfuerzo que le dedican a la misma, así como si ese rendimiento está también relacionado con las metodologías docentes. A través de un análisis ANOVA, encontramos que el rendimiento está relacionado con la percepción pero no con las metodologías novedosas introducidas a raíz de nuestra entrada en el EEES. Los resultados de este estudio nos permiten reflexionar sobre la idoneidad de las herramientas pedagógicas que actualmente utilizamos.

Palabras clave: Rendimiento académico, dirección de recursos humanos, empresas turísticas, percepción del estudiante.

Recibido: 7 de julio de 2015

Devuelto para su revisión: 17 de enero de 2016

Aceptado: 10 de marzo de 2016

* Profesora Extraordinaria en Universidad de Panamá. CIDETE Coclé (Penonomé).

Profesora en la Universidad de Cádiz, España (Excedencia).E-mail: mangele.frende@uca.es

** Departamento de Organización de Empresas. Facultad de Ciencias Sociales y de la Comunicación. Universidad de Cádiz. Avda. de la Universidad s/n. 11405 JEREZ DE LA FRONTERA, Cádiz (España). E-mail: josemaria.biedma@uca.es

*** Departamento de Estadística e Investigación Operativa. Facultad de Ciencias Sociales y de la Comunicación. Universidad de Cádiz. Avda. de la Universidad s/n. 11405 JEREZ DE LA FRONTERA, Cádiz (España). E-mail: manuel.arana@uca.es 


\title{
Factors that are determining the academic performance of students in the subject of human resource management in tourism firms
}

\begin{abstract}
This paper aims to study the factors that influence in academic performance of students in the subject of Human Resource Management in Tourism Firms from the University of Cádiz. Specifically, we analyze whether the perception on the subject is related to the effort they devote to it, and whether that performance is also related to teaching methodologies. Through an ANOVA analysis, we find that performance is related to the perception but not to the new methodologies introduced following our entry into the EHEA. The results of this study allow us to reflect on the appropriateness of the pedagogical tools we use today.
\end{abstract}

Keywords: Academic performance, human resource management, touristic firms, students' perception.

\section{INTRODUCCIÓN}

La Dirección de Recursos Humanos (DRH) es un elemento esencial en la competitividad de las empresas en general, y de las turísticas en particular. La mayor parte de las actividades de la cadena de valor de las empresas que forman parte de la industria del turismo y ocio son llevadas a cabo por personas. Hunter, Schmidt y Judiesch (1990) analizan cómo las empresas intensivas en mano de obra pueden obtener ventajas competitivas si se dispone de empleados competentes y además, se crean las condiciones adecuadas para que se traslade, de manera continuada, la productividad de los mejores empleados al resto del equipo de trabajo. Los empleados se convierten así en el principal recurso productivo y diferenciador en este tipo de organizaciones (Lillo, Ramón y Sevilla, 2007).

Esta consideración de las personas que trabajan en el sector turístico como activo clave de las empresas es aceptada comúnmente en la literatura especializada (Lillo, 2009). En esta línea, Acosta, Fernández y Mollón (2002) consideran que en el sector turístico los recursos humanos constituyen la principal ventaja competitiva. Por ello, deberían ser el centro de atención de los directivos que deseen la excelencia de los servicios que ofertan, ya que ello repercutirá positivamente en su cuenta de resultados. El compromiso, la motivación, el trabajo en equipo, entre otras variables, son elementos estratégicos que influyen en la calidad del servicio, y como consecuencia, en la satisfacción de los clientes que buscan, en las empresas turísticas y de ocio, nuevas emociones y experiencias (Heskett, Jones, Loveman, Sasse y Schilesinger, 1994).

En el ámbito empresarial también se destaca la importancia de los recursos humanos para el éxito empresarial. El Libro Blanco de Exceltur (2006) destaca la importancia de la Gestión de Recursos Humanos en el sector turístico. Concretamente, señala que dicha Gestión se ha convertido en una de las políticas claves de la empresa turística, porque tiene la responsabilidad de generar un entorno de trabajo en el que los empleados se encuentren motivados para poner su capital profesional al servicio del cliente, la empresa y el negocio. 
La DRH se convierte, por tanto, en una de las políticas claves de la empresa turística, al ser, entre otros, la responsable de generar un entorno de trabajo en el que los empleados estén dispuestos a poner su capital profesional, esto es, su conocimiento, su competencia, su talento, su ilusión y en definitiva su compromiso al servicio del cliente, la empresa y el negocio (Huang, 2000).

Todo lo anterior, demuestra la importancia del adecuado aprendizaje por parte de los estudiantes universitarios de esta materia. Ello beneficiará a las empresas donde éstos vayan a ejercer sus actividades profesionales futuras.

En los últimos años, se han realizado estudios en el ámbito de la universidad enfocada a estudiar las variables que inciden en el rendimiento académico de los estudiantes (Fernández y Alonso-Tapia, 2012). Sin embargo, existe una escasez de trabajos enfocados en la materia de DRH en las empresas turísticas. Por ello, y dada la importancia del tema, el objetivo de este trabajo es analizar los factores que influyen en el rendimiento académico de los estudiantes de esta asignatura que se imparte en el Grado de Turismo de la Universidad de Cádiz.

Los resultados de este trabajo nos permitirán diseñar, en su caso, propuestas metodológicas y de contenido que mejoren la calidad del aprendizaje de los estudiantes; siendo éste uno de los fines primordiales que se recogen en nuestras leyes universitarias (Maquillón Sánchez y Hernández Pina, 2011).

Tras esta introducción, en el siguiente epígrafe revisamos la literatura existente sobre el tema objeto de estudio y planteamos los objetivos de la investigación. En el tercer epígrafe describimos la muestra, cómo se han obtenido los datos y las técnicas estadísticas empleadas. Posteriormente analizamos los resultados y finalizamos con las conclusiones del estudio.

\section{REVISIÓN DE LA LITERATURA}

El rendimiento académico es una de las dimensiones que más influye en el aprendizaje de los estudiantes y, por tanto, en su desempeño profesional futuro. Sin embargo, no existe una definición que sea universalmente aceptada. Algunos autores se refieren a ellos como los resultados inmediatos que alcanzan los estudiantes a lo largo de su carrera, a las calificaciones obtenidas en la primera convocatoria o el haber finalizado a tiempo los estudios, entre otros (Dávila, García-Artiles, Pérez-Sánchez y Gómez-Déniz, 2015; Tejedor y García-Valcárcel, 2007).No obstante, Ocaña (2011) sostiene que las definiciones anteriores son susceptibles de ciertas dosis de subjetividad en los mecanismos de evaluación, y también se le critica la falta de homogeneidad en las formas de evaluar (Willcox, 2011). Así, otras definiciones vinculan el rendimiento académico al mundo laboral (Tejedor y García-Valcárcel, 2007), siendo la dilación de su medida una de las críticas que reciben estas propuestas.

Dada la falta de consenso, en este trabajo seguimos la propuesta realizada por Manzano (2007), que lo define como el esfuerzo y la capacidad de trabajo que el estudiante aplica en su proceso de aprendizaje; es decir, la disposición y el interés del estudiante por la materia. En este caso, las horas de estudio pueden ser un predictor del resultado académico (Tejedor y García-Valcárcel, 2007). Esto es apoyado por otros autores, quienes indican 
como variables proxys del rendimiento la dedicación fuera del aula, los hábitos de estudio y la responsabilidad (Hernández-Pina, García-Sanz et al., 2002; Elousa, López-Jáuregui y Mújica, 2012; Yu, 2011).

En lo que sí parece haber unanimidad es sobre la incidencia del rendimiento académico sobre el éxito profesional. Dada su importancia, no es de extrañar la cantidad de trabajos que se han ocupado de medirlo, de mejorarlo y de analizar los factores que influyen en él (Biggs, 1993; Edel-Navarro, 2003; Izar-Landeta, Yazunza y López-Gama, 2011). Dentro de ésta última línea de investigación, se considera que, entre otros, las metodologías docentes y la percepción que los estudiantes tienen de la asignatura influye en el esfuerzo destinado a su aprendizaje, y como consecuencia, en su aprendizaje (Alfalla-Luque, Medina-López y Arenas-Márquez 2011; Fish, 2008; Pal y Busing, 2008; Rinaudo, Chiecher y Donolo, 2003).

Con la incorporación de España en el EEES, las universidades introdujeron metodologías docentes más novedosas respeto a las existentes, con el fin de mejorar el aprendizaje de los estudiantes, de forma que puedan convertirse en profesionales capaces de desempeñar adecuadamente las tareas que sus ocupaciones futuras les demanden. En esta línea, Dávila et ál., (2015) proponen, además de las clases magistrales, los seminarios-talleres, clases prácticas, prácticas externas, tutorías, estudio y trabajo en grupo, y estudio y trabajo individual, como modalidades "nuevas" dentro de este marco de enseñanza centrada en la actividad activa del alumnado. En este sentido se asume que, además de otros factores, el uso de determinadas metodologías tiende a favorecer la visión de los alumnos por la materia y despierta más su interés (Rinaudo et al., 2003).

Sin embargo, las conclusiones a las que llegan varios trabajos sobre la existencia de una relación positiva entre las metodologías "activas" empleadas y el rendimiento académico son contradictorias. Estudios como los de, por ejemplo, Alfalla-Luque et al. (2011) y Ullah, Sagheer, Sattar y Khan (2013) ponen de manifiesto la relación positiva entre esas metodologías "nuevas" y el rendimiento académico de los estudiantes, medido éste por la adquisición de competencias. De igual forma, Álvarez, García y Gil (1999) y Medina (2001), afirman que la relación entre metodología y rendimiento es clara, porque consideran que la primera incide en la optimización del rendimiento del alumnado universitario. En este sentido, dichos autores apuntan la importancia y la necesidad de una enseñanza que, entre otras cuestiones, impulse la exposición de distintos puntos de vista, promueva la reflexión y la solución de problemas. Fernández (2006) ha establecido también una relación entre metodología de enseñanza y mejora de resultados, considerando que la aplicación de métodos con participación del alumno conlleva aprendizajes más intensos y duraderos. En la misma línea, se puede citar a Jonassen, Howland, Marra y Crismond (2008) y el estudio de Chou y Chen (2008) realizado con estudiantes de Tecnología y Gestión de la Información de la Universidad de Pennsylvania, que apoyan la mejora de resultados académicos con el uso de la plataforma wiki, como modalidad de aprendizaje cooperativo.

Más recientemente, en un estudio realizado con alumnos de la asignatura Informática I del Grado en Informática de la Universidad de Valencia, que combinaba el uso de los principios del aprendizaje cooperativo, el aprendizaje basado en problemas y la técnica del aprendizaje mediante la explicación, se evidenció una correlación entre 
la metodología docente y la mejora del rendimiento académico (Grimaldo-Moreno y Arevalillo-Herráez, 2011). Así, parece que la metodología de enseñanza está considerada como una de las variables pedagógicas explicativas del rendimiento (Tejedor y GarcíaValcárcel, 2007).

Por otra parte, es posible encontrar trabajos en los que los autores no encuentran relación alguna entre dichas variables. En un estudio realizado por Florido, Jiménez y Santana (2011) sobre el impacto de la metodología de seguimiento del alumno (evaluación continua) en el rendimiento de éste concluyó que dicha metodología no mejoraba los resultados respecto a la utilizada con anterioridad (metodología tradicional).Concretamente, los alumnos pertenecían a la Universidad de Las Palmas de Gran Canaria y cursaban la asignatura de Economía Mundial. En la misma línea, una investigación realizada con alumnos de la Universidad de Santiago de Compostela de la asignatura de Toxicología de la Licenciatura en Farmacia, que combinaba la utilización de la metodología docente tradicional (clase magistral) con las nuevas metodologías (trabajo cooperativo, uso de plataformas docente, etc.), concluyó que los resultados académicos no mejoraban sustancialmente con relación a los obtenidos en cursos anteriores, en los que se utilizaba únicamente la metodología tradicional (Cruz, López-Rivadulla, Quintela, De Castro y Concheiro, 2009).

Estos resultados fueron corroborados también por, Tejedo, Pontones, Ramírez y Tejada (2015). Éstos demostraron la nula incidencia del uso de metodologías "activas" en la materia de contabilidad financiera. Igualmente, Leeds, Stull y Westbrook (1998) encontraron que las metodologías activas no mejoraron los resultados de los estudiantes de contabilidad, pero sí con la lección magistral. Parece ser, que las técnicas de enseñanza más participativas exigen más dedicación por parte de los estudiantes y que, por tanto, prefieren seguir siendo meros receptores de información tal como hacen con la clase magistral y sólo tener que trabajar para el examen (Cruz et al., 2005).

Ante la inconsistencia de los resultados encontrados, nos preguntamos si en los estudiantes de la asignatura de Dirección de Recursos Humanos en Empresas Turísticas (DRHET) que se imparte en el Grado de Turismo de la Universidad de Cádiz, la utilización de determinadas metodológicas "nuevas", comunes a las que se emplea en otras materias de la propia universidad, tienen incidencia en sus resultados académicos.

Por otra parte, la percepción de los estudiantes por la materia también afecta a su proceso de aprendizaje (Wolters y Pintrich 1998). Como señalan McRobbie y Tobin (1997), si los contenidos son percibidos como interesantes, importantes o útiles, los estudiantes están más dispuestos a aprender. Uno de los factores que más influyen en la percepción es la utilidad real que ven en la asignatura (Ausnubel, 1989). Igualmente, la visión que tenga el estudiante de la tarea a realizar y las metas que pretenden alcanzar constituyen factores que guían y dirigen la conducta del estudiante en el ámbito académico (Maquillón y Hernández-Pina 2011).Por otra parte, Huertas y Agudo (2003) argumentan que los estudiantes sólo se esfuerzan cuando consideran que la asignatura es relevante para su desempeño profesional.

En el curso académico 2013/2014 se introdujeron nuevos contenidos en la asignatura que son de interés en el sector turístico empresarial. Entre éstos se incluyen la gestión de personal socialmente responsable, la utilización de las redes sociales en la dirección de recursos humanos, así como la importancia de la inteligencia emocional y la creatividad. 


\subsection{Objetivos}

Tras la revisión teórica, podemos extraer la importancia de estudiar los factores que inciden en el rendimiento académico. Aunque se han hecho muchos avances, existen muy pocos trabajos que analicen la relación entre la metodología empleada y la percepción que el estudiantes tiene sobre la asignatura, y más específicamente, en lo que se refiere a la materia de Dirección de Recursos Humanos de las Empresas Turísticas. Por ello, en este trabajo nos proponemos dar respuesta a las dos cuestiones siguientes:

- ¿existe relación entre la percepción de la asignatura y el rendimiento académico?

- ¿existe relación entre las metodologías "nuevas" empleadas y el rendimiento académico?

\section{METODOLOGÍA DE LA INVESTIGACIÓN}

Para dar respuesta a las preguntas de investigación anteriormente planteadas nos hemos centrado en los estudiantes del Grado en Turismo de la Universidad de Cádiz que se imparte la Facultad de Ciencias Sociales y de la Comunicación, ubicada en el campus de Jerez. Esta titulación se imparte como diplomatura desde el año 2004 y fue pionera en España en su adecuación al EEES desde el curso académico 2003-2004 como experiencia piloto de implantación al crédito europeo.

Los estudiantes del Grado de Turismo representan un 28,3\% del total de alumnos que cursan estudios de grados en la facultad en este cursos académico. La demanda de los estudios turísticos es muy alta, siendo el número de solicitudes de nuevo ingreso presentadas de 220, y el de admitidos de 146, con una nota media de selectividad de 6,91. Esta demanda no es de extrañar por la importancia que tiene la industria turística y de ocio en la Provincia de Cádiz, siendo éste uno de las fuentes importantes de ingreso de la zona y que supone, según datos de INE en el 2009, del 70\% del PIB.

\subsection{Muestra y fuente de datos}

Este trabajo se ha llevado a cabo durante el curso académico 2011/2012 en la asignatura de DRHET que se imparte en el segundo semestre, del segundo curso del grado de Turismo de la Universidad de Cádiz. El total de alumnos matriculados es esta materia es de 120 .

Para obtener la información de las variables objeto de estudio se diseñó dos cuestionarios: uno de ellos recogía las preguntas relativas a la percepción de la materia en cuanto a la importancia y utilidad que ellos le daban para su futuro profesional y debían puntuar sobre una escala Likert-5, donde 1 representaba "totalmente en desacuerdo" y 5 "totalmente de acuerdo". Tuvieron un máximo de 10 minutos para completarlo y se les pasó a los estudiantes a las dos semanas de comienzo del semestre, para evitar sesgos posteriores relacionados con el profesor $\mathrm{u}$ otros aspectos.

El otro cuestionario se les pasó dos semanas antes de finalizar el semestre y recogía preguntas relativas, por un lado, a su opinión sobre la utilización de determinadas 
metodologías "nuevas" empleada, tales como: el estudio de casos, trabajo cooperativo y trabajo en grupo. Igualmente, tenían que puntuarlas sobre una escala Likert-5.

Por otro lado, el cuestionario también incluyó una pregunta dedicada al "tiempo de estudio" con objeto de medir la variable "rendimiento académico". La cantidad de horas es considerada como un potente predictor del éxito académico. Se considera que cuanto mayor es el esfuerzo destinado a una asignatura, mayor será su proceso de aprendizaje (Astin, 1999). Por ello, se les pidió a los estudiantes que señalasen el tiempo que cada semana le dedicaban al estudio de la asignatura: menos de dos horas, entre dos y cuatro horas y más cuatro horas, siendo éste el tiempo considerado en la guía de la misma como el mínimo adecuado para un aprendizaje óptimo.

La información suministrada por los alumnos en los dos cuestionarios era anónima y se les pidió total sinceridad en sus respuestas.

La población de este estudio estaba compuesta por 120 estudiantes. Sin embargo, los que asistían habitualmente a clases eran 75, que fueron los que completaron ambos cuestionarios. La tasa de participación es de un 62,5\%, de una edad media de 21,75 años, siendo la presencia masculina $(34,25 \%)$ bastante inferior a la femenina. Esta proporción se corresponde con la presencia mayoritaria de las mujeres en el Grado en Turismo de la Universidad de Cádiz (aproximadamente 68,30\%). La tabla 1 muestra la ficha técnica del estudio.

\section{Tabla 1}

\section{FICHA TÉCNICA DEL ESTUDIO}

\begin{tabular}{|c|l|}
\hline UNIVERSO & Titulación de Turismo de la Universidad de Cádiz \\
\hline ÁMBITO GEORGRÁFICO & Facultad de CC.SS. y de la Comunicación \\
\hline ELEMENTO MUESTRAL & Estudiantes matriculados en la asignatura de DRHET \\
\hline TAMAÑO MUESTRAL & 120 \\
\hline DISEÑO MUESTRAL & Diseño del cuestionario \\
\hline INFORMACIÓN RECOGIDA & $\begin{array}{l}\text { Percepción de la materia, metodología empleada y } \\
\text { horas de estudio }\end{array}$ \\
\hline
\end{tabular}

\subsection{Técnicas de análisis}

Para responder a los objetivos planteados, se comenzó con un análisis exploratorio de diagramas de cajas y bigotes. Este análisis proporciona una primera aproximación al comportamiento de las variables objeto de estudio y sus posibles relaciones. En consecuencia, nos ha orientado sobre la selección y aplicación de las técnicas estadísticas adecuadas: por un lado, y para el estudio de la relación entre dos variables, nos hemos apoyado en regresiones, así como en los contrastes de hipótesis para el caso lineal y ordinal (Spearman), entre otros. Para los detalles sobre estos diagramas y técnicas se recomienda al lector los trabajos de Uriel y Aldás (2005) y de Alegre, Cladera y Juaneda (2003).

En la aplicación de estas técnicas se ha utilizado el paquete estadístico Statgraphics Centurion XVII. 


\section{RESULTADOS}

Para responder a la primera cuestión planteada en este trabajo sobre la existencia de relación entre la percepción que tienen sobre la materia y el rendimiento académico de los estudiantes, comenzamos con un análisis exploratorio que nos permite un primer acercamiento a las posibles relaciones entre ambas variables. Para ello, nos ayudamos de un diagrama de cajas y bigotes para la variable Percepción por cada valor del rendimiento académico (Figura 1).

La caja, representada por un rectángulo, aglutina al 50\% de los valores de la variable percepción; más concretamente, los valores comprendidos entre el primer y el tercer cuartil. La caja aparece dividida por un segmento que representa la mediana. Los valores por debajo del primer cuartil se corresponden con el segmento ('bigote') a la izquierda de la caja; y los valores por encima, con el segmento a la derecha. La longitud de cada 'bigote' no puede superar 1,5 veces la longitud de la caja. Si se queda algún valor fuera del correspondiente 'bigote', éste aparece representado con un símbolo (cuadrado, estrella,...), tal como ocurre para el segundo valor de rendimiento académico (Horas de estudio).

\section{Figura 1}

\section{GRÁFICO DE CAJAS Y BIGOTES}

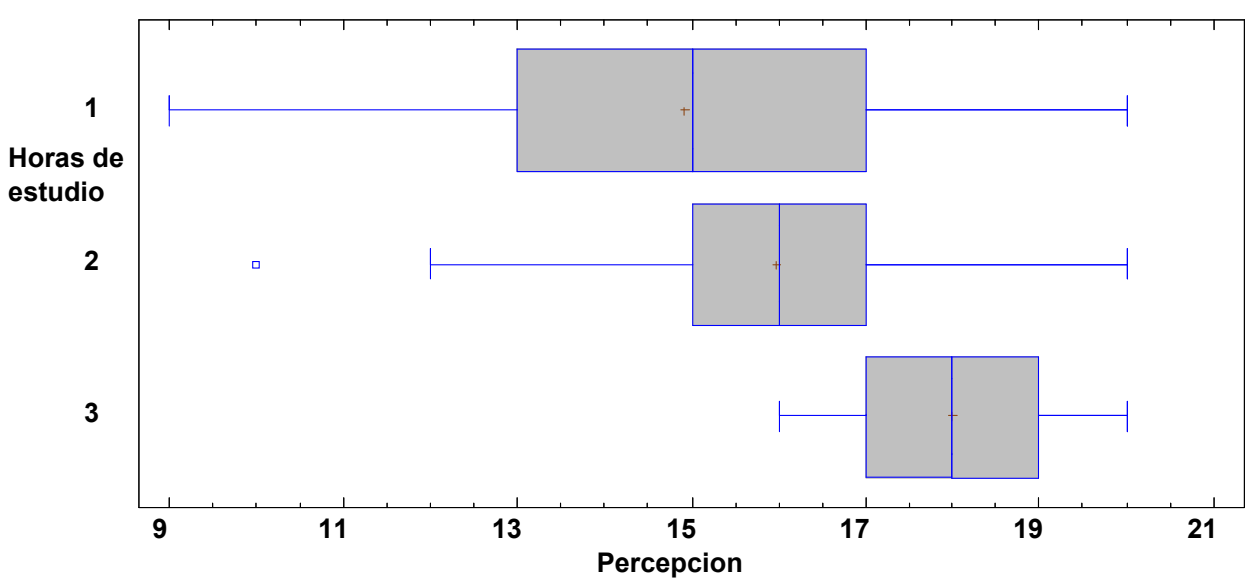

Como se puede ver en la Figura 1, las cajas van quedando desplazadas a la derecha conforme aumenta el rendimiento académico, por lo que todo apunta a una vinculación entre la percepción y el rendimiento académico (RA). En particular, a mayor percepción encontramos un mayor rendimiento académico, que marcará nuestra línea de trabajo para ratificar esta situación más adelante. De forma detallada, los diferentes promedios de percepción en los diferentes rangos del rendimiento académico son los siguientes (ver tabla 2): 
Tabla 2

PROMEDIOS DE PERCEPCIÓN

\begin{tabular}{|c|c|l|}
\hline$R A$ & Recuento & \multicolumn{1}{|c|}{ Promedio } \\
\hline 1 & 26 & 14,9231 \\
\hline 2 & 39 & 15,9744 \\
\hline 3 & 6 & 18,0 \\
\hline Total & 71 & 15,7606 \\
\hline
\end{tabular}

Para comprobar la posible relación existente entre ambas variables realizamos, en primer lugar, una regresión lineal, cuyos resultados se recogen en la tabla 3.

Tabla 3

CORRELACIONES ENTRE PERCEPCIÓN Y (RA)

\begin{tabular}{|l|r|r|}
\cline { 2 - 3 } \multicolumn{1}{c|}{} & \multicolumn{1}{c|}{ Percepción } & \multicolumn{1}{c|}{ RA } \\
\hline Percepción & & 0,3468 \\
& & P-Valor $=0,0030$ \\
\hline \multirow{2}{*}{ RA } & 0,3468 & \\
\hline
\end{tabular}

Observamos que el coeficiente de correlación lineal es 0,3468, muy alejado del 1, lo que indica una escasa relación lineal entre percepción y rendimiento académico. Esta observación queda refrendada por un p-valor de 0,0030 .

En segundo lugar, y como medición alternativa al ajuste, podemos contar con la correlación ordinal de Spearman (tabla 4).

Tabla 4

CORRELACIÓN ORDINAL DE SPEARMAN

\begin{tabular}{|l|r|r|}
\cline { 2 - 3 } \multicolumn{1}{c|}{} & \multicolumn{1}{c|}{ Percepción } & \multicolumn{1}{c|}{ RA } \\
\hline Percepción & & 0,3315 \\
RA & 0,3315 & \\
& & \\
\hline & p-valor $=0,0055$ \\
\hline
\end{tabular}

Del mismo modo, obtenemos un coeficiente de Correlación de 0,3315 , lo cual indica la escasa relación lineal existente, con un p-valor de 0,0055. Ninguno de los modelos alternativos de regresión supera un coeficiente adecuado para admitir una relación entre las dos variables. Es por ello que un análisis de asociación lineal entre rendimiento académico y percepción no es aconsejable. 
Para proceder, en casos como el anterior, con el estudio de la influencia de las categorías en las que se encuentra una variable sobre otra, hemos realizado el Análisis de la Varianza (ANOVA). Previamente al mismo, se han realizado los contrastes asociados a la verificación de las hipótesis de ANOVA. Para la normalidad, se ha usado el contraste de Kolmogorov-Smirnov y el de Shapiro-Wilk. Para la verificación de la igualdad de varianzas, la prueba de Levene's. Para la comparación de dos poblaciones, bajo normalidad, se ha utilizado la prueba F para la comparación de varianzas, y la prueba t para la comparación de medias.

Siguiendo con el caso que nos ocupa con rendimiento académico y percepción, pasamos a un análisis de la varianza ANOVA (ver tabla 5) para comprobar esa posible vinculación que se desprende de la figura 1 como resultado del análisis exploratorio. Hemos comprobado que las hipótesis previas son satisfechas (normalidad, homocedasticidad e independencia) por lo que procedemos a su aplicación.

Tabla 5

ANOVA PARA PERCEPCIÓN POR RA

\begin{tabular}{|l|l|l|l|l|l|}
\hline \multicolumn{1}{|c|}{ Fuente } & \multicolumn{1}{c|}{$\begin{array}{c}\text { Sumade } \\
\text { Cuadrados }\end{array}$} & Gl & Cuadrado Medio & \multicolumn{1}{|c|}{ Razón-F } & Valor-P \\
\hline Entre grupos & 50,1091 & 2 & 25,0545 & 5,00 & 0,0094 \\
\hline Intra grupos & 340,821 & 68 & 5,01207 & & \\
\hline Total (Corr.) & 390,93 & 70 & & & \\
\hline
\end{tabular}

La ANOVA arroja un p-valor muy bajo de 0,0094 lo que implica que existe una diferencia estadísticamente significativa entre Percepción y nivel de rendimiento académico. Es decir, existe vinculación entre percepción y rendimiento académico. No obstante, y para profundizar en esta diferencia, aplicamos un análisis más detallado comparando niveles, que según la Figura 1 encontraremos fundamentalmente entre el nivel 1 y el 3 de rendimiento académico. Para ello, aplicamos Pruebas de Múltiples Rangos para Percepción por Rendimiento académico, como puede observarse en las tablas 6a y 6 b.

Tabla 6a

\begin{tabular}{|l|l|l|l|}
\hline Nivel & Casos & Media & Grupos Homogéneos \\
\hline 1 & 26 & 14,9231 & $\mathrm{vX}$ \\
\hline 2 & 39 & 15,9744 & $\mathrm{vXX}$ \\
\hline 3 & 6 & 18,0 & $\mathrm{v} X$ \\
\hline
\end{tabular}

Método: 95,0 porcentaje Bonferroni. 
Tabla 6b

\begin{tabular}{|l|l|l|l|}
\hline Contraste & Sig. & Diferencia & + /- Limites \\
\hline $1-2$ & & $-1,05128$ & 1,39135 \\
\hline $1-3$ & $*$ & $-3,07692$ & 2,48892 \\
\hline $2-3$ & & $-2,02564$ & 2,40988 \\
\hline
\end{tabular}

* indica una diferencia significativa.

Como se desprende de los datos anteriores, existe una diferencia significativa entre la percepción obtenida para los rendimientos académicos 1 y 3 . No se puede derivar de la aplicación de esta prueba una diferencia significativa entre la percepción entre 1 y 2 , así como entre 2 y 3 . No obstante, podemos profundizar en un estudio más detallado y comparativo entre estos niveles dos a dos, en particular entre los que tenemos dudas, como son las comparativas con el nivel 2. Para ello, procedemos con una comparación de Percepción dos a dos, por niveles:

Asumimos que Percepción proviene de distribuciones normales, ya que los contrastes de normalidad aplicados arrojan p-valores inferiores al 0,05. Por tanto, vamos a realizar las comparaciones paramétricas para la media mediante el contraste o prueba t. Comenzamos con los dos primeros niveles para la variable rendimiento académico (Figura 2).

\section{Figura 2 \\ GRÁFICO DE CAJAS Y BIGOTES}

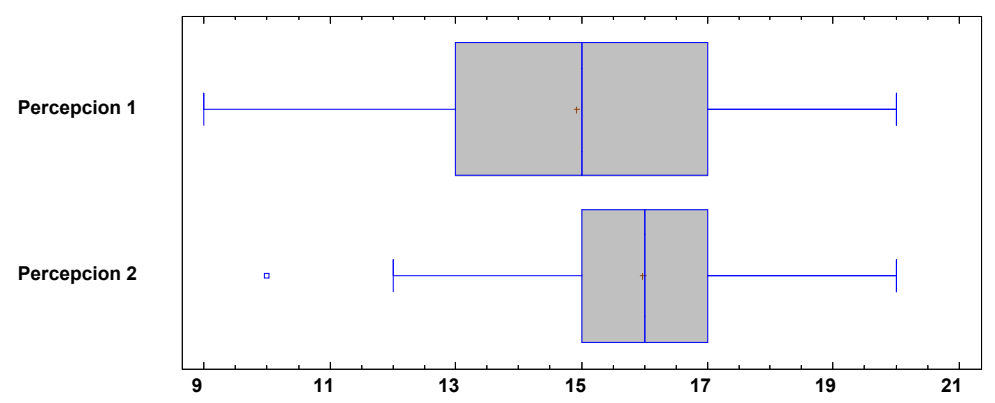

A la vista de la figura anterior, podemos pensar que la percepción media con un rendimiento académico de 1 es menor que con un rendimiento académico de 2. Para corroborarlo procedemos a aplicar el correspondiente contraste t. Previamente hemos contrastado las varianzas mediante un contraste $\mathrm{F}$ de lo que se ha derivado que no podemos suponer igualdad de varianzas. Utilizamos esta información para el siguiente contraste:

Hipótesis nula: media $1=$ media2

Hipótesis Alt.: media1 < media2

$\mathrm{t}=-1,67381$ valor- $\mathrm{P}=0,0510763$

No se rechaza la hipótesis nula para alfa $=0,05$. 
Por tanto, con un 5\% de nivel de significación, no podemos rechazar la igualdad de medias y asumir que la media en nivel 1 de Percepción es menor que en el nivel 2 de rendimiento académico. Es decir, no tenemos evidencias para determinar que aquellos estudiantes con niveles de percepción 1 y 2 vayan a tener una vinculación distinta en su rendimiento académico sino que más bien éste será semejante.

La Figura 3 recoge el análisis exploratorio realizado para los dos niveles más alto de rendimiento académico $(\mathrm{P} 2$ y $\mathrm{P} 3)$.

Figura 3

GRÁFICO DE CAJAS Y BIGOTES

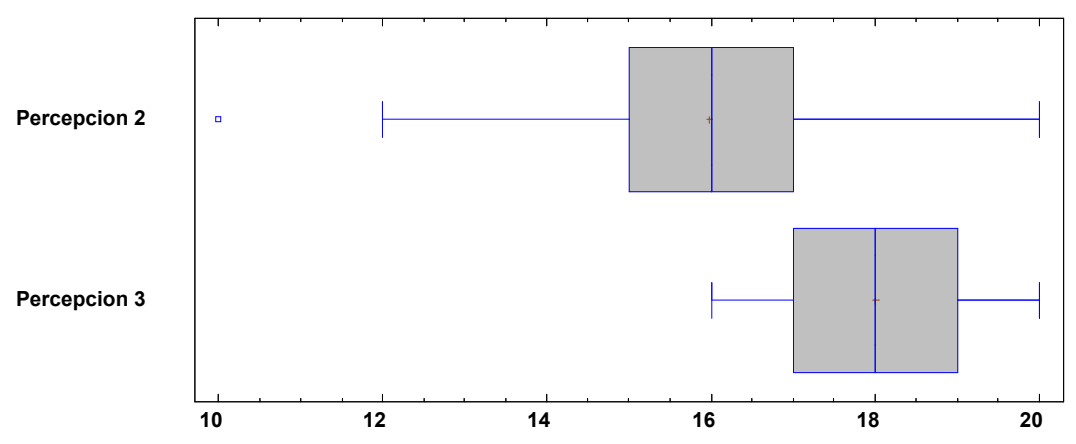

A partir de la Figura 3, intuimos una diferencia clara en las percepciones medias de los dos rendimientos académicos más altos. Procediendo como con anterioridad, constatamos que podemos asumir igualdad en las varianzas de percepción a partir del contraste $\mathrm{F}, \mathrm{y}$ procedemos al contraste t correspondiente.

Hipótesis nula: media $1=$ media2

Hipótesis Alt.: media1 < media2

$\mathrm{t}=-2,5511$ valor- $\mathrm{P}=0,00718937$

Se rechaza la hipótesis nula para alfa $=0,05$.

A la vista de los resultados anteriores podemos concluir que, de forma significativa, la percepción media en cada uno de los dos niveles superiores de rendimiento académico (P2 y P3) es diferente. Así, podemos considerar que el rendimiento académico más alto y el intermedio reciben diferente percepción, siendo más alta cuanto mayor es el rendimiento académico.

En cuanto a la segunda cuestión planteada en esta investigación, que pretende conocer si existe relación entre las metodologías empleadas y el rendimiento académico, procedemos de la misma forma que anteriormente. Así, comenzamos con un análisis exploratorio mediante el diagrama de cajas y bigotes para las variables metodologías y rendimiento académico (Figura 4). 
Figura 4

GRÁFICO DE CAJAS Y BIGOTES

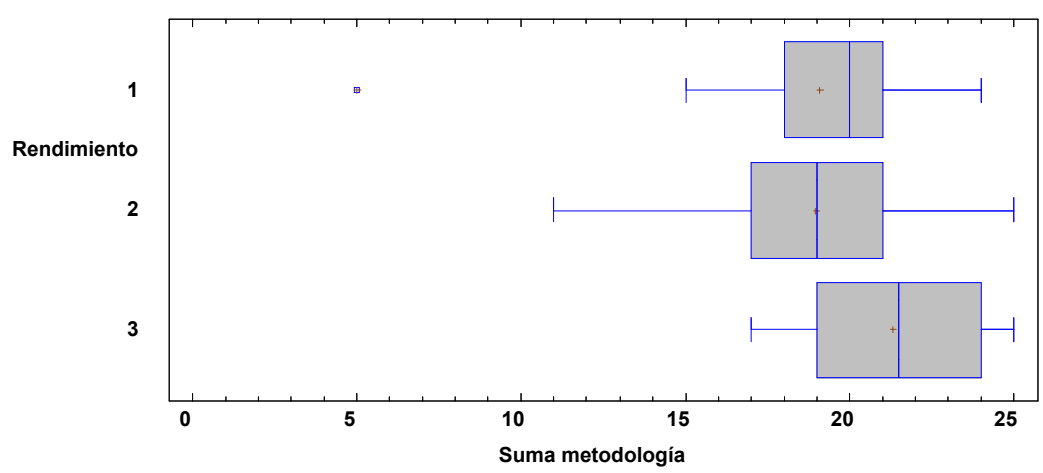

De la observación de esta figura se desprende, a modo intuitivo, una escasa diferencia en el comportamiento de la metodología frente al rendimiento. Es decir, intuimos que no existe una relación clara o significativa entre ambas variables. Para ello, y una vez comprobado que las hipótesis previas (normalidad, homocedasticidad e independencia), procedemos con un ANOVA, cuyos resultados se recogen en la tabla 7.

Tabla 7

ANOVA PARA METODOLOGÍA Y RA

\begin{tabular}{|c|c|c|c|c|c|}
\hline Fuente & $\begin{array}{c}\text { Suma de } \\
\text { Cuadrados }\end{array}$ & Gl & Cuadrado Medio & Razón-F & Valor-P \\
\hline Entre grupos & 29,6771 & 2 & 14,8386 & 1,42 & 0,2495 \\
\hline Intra grupos & 712,154 & 68 & 10,4729 & & \\
\hline Total (Corr.) & 741,831 & 70 & & & \\
\hline
\end{tabular}

Como se desprende de la tabla anterior ( $p$-valor $=0,2495$ ), podemos asumir que no hay relación entre ambas variables, no viéndose influenciada o afectada la metodología por el rendimiento académico.

\section{DISCUSIÓN Y CONCLUSIONES}

En este trabajo hemos tratado de determinar las variables que influyen en el rendimiento académico de los estudiantes de la asignatura de DRHET del Grado en Turismo de la Universidad de Cádiz. Específicamente, hemos analizado si existe relación entre la percepción que los estudiantes tienen por la materia y su resultado de aprendizaje, así también como las metodologías empleadas por el profesor. 
Con relación a la primera cuestión planteada en nuestro trabajo, podemos afirmar que la percepción que tienen los estudiantes de la asignatura DRHET está vinculada al rendimiento académico, medido por las horas de estudio que le dedican a la misma (Manzano, 2007). En concreto, encontramos diferencias significativas entre la percepción y los niveles bajos y altos de rendimiento. Es decir, que cuando los estudiantes tienen una baja visión de la importancia de la asignatura, el tiempo que le dedican es también reducido, lo que redunda en un aprendizaje menor. Igualmente, ocurre cuando la percepción es alta: en este caso, el rendimiento es mayor. Sin embargo, no encontramos evidencias de diferencias entre la percepción cuando el esfuerzo (horas dedicadas) es medio. Así, en estudios posteriores se podría eliminar este nivel de esfuerzo, y considerar sólo dos medidas: baja y alta.

No obstante, podemos afirmar que existe un vínculo entre la percepción y el rendimiento académico. Estos resultados son consistentes con los obtenidos en otros trabajos. Así, Alfalla-Luque et al. (2011) encontraron que la percepción de los estudiantes de la asignatura Dirección de Operaciones condiciona su proceso de aprendizaje. Como indican Siegle y McCoach (2005), la visión que ellos tengan de la utilidad de una materia para su futuro profesional (recompensas extrínsecas) influye en el esfuerzo que le dedican a la misma. Igualmente, Astin (1999) señala que para alcanzar los objetivos recogidos en un programa educativo en concreto, los estudiantes deben dedicarle un nivel de esfuerzo determinado, que va en relación a la importancia que ellos perciban del mismo.

En cuanto al vínculo entre la metodología y el rendimiento académico, no encontramos una relación estadísticamente significativa. Es decir, no hemos podido probar estadísticamente que la utilización de metodologías nuevas afecta al esfuerzo que los estudiantes le dedican a la asignatura de DRHET. Parece que estas metodologías, las cuales suponen más esfuerzo por parte del estudiante que la típica lección magistral, tienden a desmotivarlos y surte el efecto contrario, dedicándoles así menos tiempo de estudio (Cruz et al.,2009). Igualmente, estos resultados se encuentran en la línea de los obtenidos por RodríguezMartín y Álvarez-Arregui (2013), quienes no detectaron variaciones en el aprendizaje de los estudiantes por la introducción de nuevas metodologías a raíz de la incorporación de las universidades españolas al Plan Bolonia. De hecho, los estudiantes opinan que "es un continuismo metodológico que en muchos casos se ha traducido en un empobrecimiento de la docencia..." (Rodríguez-Martín y Álvarez-Arregui, 2013:110). También, AlfallaLuque et al. (2011), en su estudio centrado en la materia de Dirección de Operaciones, no obtuvieron resultados significativos entre las metodologías "novedosas" y las calificaciones obtenidas en las pruebas de conocimiento. En la misma línea, el trabajo de Leeds et al. (1998) que con la lección magistral (metodología tradicional) los estudiantes obtuvieron mejores resultados que cuando se utilizaban otros métodos más novedosos, tales como el trabajo cooperativo, trabajo en grupo, etc.

En este trabajo sólo hemos considerado dos de los varios factores que influyen en el rendimiento académico. Así, en estudios futuros podríamos incluir otras variables, tales como las demográficas, cognitivas y sociales (Csikszentmihalyi, Rathunde y Whalen, 1993; Monroy y Hernández-Pina, 2014; Tomás-Miquel, Expósito-Langa y SempereCastelló, 2014) que han sido analizadas en la literatura, y ver si tienen alguna relación con el esfuerzo que los estudiantes le dedican a DRHET y, por tanto, a su aprendizaje. 
También, este estudio puede replicarse en otras materias de Recursos Humanos que se imparten en otros Grados de la Universidad de Cádiz, tal como el de Administración y Dirección de Empresas, ya que estas asignaturas tienen estructuras distintas y difieren en algunas de las metodologías empleadas.

No obstante, los resultados de este trabajo son interesantes para hacernos reflexionar sobre cómo mejorar el rendimiento de los estudiantes de DRHET, ya que como señalan Siegle, Rubensteiny Mitchell (2014), el esfuerzo de los estudiantes en las materias que cursan suelen ser entre un 10\%-50\% por debajo de su capacidad, lo que influye en su proceso de aprendizaje y, por tanto, en su éxito profesional. En esta línea, los profesores deberíamos hacer un esfuerzo en hacer más atractiva y útil las asignaturas y comunicarles explícitamente la importancia de la misma para su desarrollo profesional y para la competitividad de las empresas y organizaciones en las que trabajarán.

Para ello, habrá que analizar las metodologías que les son más motivadoras y que, por tanto, harán que en un futuro sean buenos directores de recursos humanos, ya que en las empresas turísticas en concreto, los empleados son el activo más preciado en todos los escalones jerárquicos y áreas de la empresa.

\section{REFERENCIAS}

ACOSTA, A.J., FERNÁNDEZ, N y MOLLÓN, M. (2002): Recursos Humanos en empresas de turismo y hostelería, Prentice Hall, Madrid.

ALEGRE, J., CLADERA, M. y JUANEDA, C. (2003): Análisis cuantitativo de la actividad turística, Ediciones Pirámides, Madrid.

ALFALLA-LUQUE, MEDINA-LÓPEZ, C. y ARENAS-MÁRQUEZ, F.J. (2011): “Mejorando la formación en Dirección de Operaciones: la visión del estudiante y su respuesta ante diferentes metodologías docentes",Cuadernos de Economía y Empresa, vol. 14, (1), pp. 40-52.

ÁLVAREZ, V., GARCÍA, E. y GIL, J. (1999): "El rendimiento académico en la Universidad desde la perspectiva del alumnado”, Revista Española de Orientación y Psicopedagogía, vol. 17, nº10, pp. 23-42.

ASTIN, A.W. (1999): Student Involvement: A Developmental Theory for Higher Education,Journal of College Student Development, Sep/Oct, pp. 518-529.

AUSUBEL, D.P. (1989): Psicología educativa: un punto de vista cognitivo. Ed. Trillas, México.

BIGGS, J. (1993):“What do inventions of students' learning processes really measure? A theoretical review and clarification, British Journal of Educational Psychology", 63, pp. 1-17.

CHOU, P.N. y CHEN, H.H. (2008): "Engagement in Online Collaborative Learning: A Case Study Using a Web 2.0 Tool”, MERLOT Journal of Online Learning and Teaching, vol. 4, pp. 574-582.

CRUZ, A., LÓPEZ RIVADUllA, M., QUINTELA, O., DE CASTRO, A. y CONCHEIRO, M. (2009): "Introducción de nuevas metodologías docentes. Influencia en el rendimiento académico y en la satisfacción del alumnado",Edusfarm, vol. 5, pp. 112-124. 
CSIKSZENTMIHALYI, M., RATHUNDE, K. y WHALEN, S. (1993): Talented Teenagers: The roots of success and failure.Cambridge, England: Cambridge UniversityPress.

DÁVILA, N., GARCÍA-ARTILES, M.D., PÉREZ SÁNCHEZ, J.M. y GÓMEZ-DÉNIZ, E. (2015): "Un modelo de regresión logística asimétrico que puede explicar la probabilidad de éxito en el rendimiento académico", Revista de Investigación Educativa, vol. 33, no 1 , pp. 27-45.

EDEL-NAVARRO, R. (2003): "El rendimiento académico: concepto, investigación y desarrollo", Revista Electrónica Iberoamericana sobre Calidad, Eficacia y Cambio en Educación, vol. 1, n ${ }^{\mathrm{o}} 2$, pp.1-15.

ELOSUA, O., LÓPEZ JÁUREGUI, A. y MÚJICA, J. (2012): “Actitudes hacia el análisis de datos: naturaleza y medida”. Revista de Psicodidáctica, vol. 17, n 2, pp.361-375.

EXCELTUR (2006): Libro Blanco de los Recurso Humanos del turismo en España. Competitividad turística y diferenciación a través de las personas, Madrid, Exceltur, Alianza para la Excelencia Turística.

FERNÁNDEZ MARCH, A. (2006): "Metodologías activas para la formación de competencias". Educatio siglo XXI, 24, pp. 35-56.

FERNÁNDEZ, C. y ALONSO-TAPIA, J. (2012): “¿Cómo motivan a los estudiantes de Ingeniería las distintas pautas de actuación docente?”, Revista Educativa Helaremos, vol.12, pp. 23-33.

FISH, L. (2008): "Graduate student project: employer operations management analysis", Journal of Education for Business, vol. 84, pp. 18-30.

FLORIDO, C., JIMÉNEZ, J.L. y SANTANA, I. (2011): “Obstáculos en el camino hacia Bolonia: efectos de la implantación del Espacio Europeo de la Educación Superior (EEES) sobre los resultados académicos”. Revista de Educación, vol.354, pp.629-656.

GRIMALDO-MORENO, F. y AREVALILLO-HERRÁEZ, M. (2011): “Metodología Docente Orientada a la Mejora de la Motivación y Rendimiento Académico Basada en el Desarrollo de Competencias Transversales", IEEE-RITA, vol. 6, (2), pp. 70-77.

HERNÁNDEZ PINA, F., GARCÍA SANZ, M. P., MARTÍNEZ CLARES, P., HERVÁS AVILÉS, R. M. y MAQUILÓN SÁNCHEZ, J. J. (2002): “Consistencia entre motivos y estrategias de aprendizaje en estudiantes universitarios", Revista de Investigación Educativa, vol. 20, $\mathrm{n}^{\circ}$ 2, pp. 487-510.

HESKETT J.L., JONES T.O., LOVEMAN G.W. , SASSER W.E. , JR. , AND SCHLESINGER L. A. (1994): "Putting the Service-Profit Chain to Work", Harvard Business Review, March-April, pp. 164-175

HUANG, T.C. (2000):“Are the Human Resources Practices of Effectives Films Disctinly Different from Those of Poorly Performing ones?", International Journal of Human Resource Management, vol. 11, $\mathrm{n}^{\circ}$ 2, pp. 436-451.

HUERTAS, J. A. y AGUDO, R. (2003): "Concepciones de los estudiantes universitarios sobre la motivación”, en C. Monereo y J. I. Pozo (Eds.) La universidad ante la nueva cultura educativa. Enseñar y aprender para la autonomía, Barcelona. Síntesis

HUNTER, J.E., SCHMIDT, F.L. y JUDIESCH, M.K. (1990): “Individual Differences in output variability as a Function of Job Complexity”, Journal of Applied Psychology, vol. $75, \mathrm{n}^{\circ} 1$, pp. 28-42. 
IZAR-LANDIETA, J., YAZUNZA CORTÉS, C. y LÓPEZ-GAMA, H. (2011): “Factores que afectan al desempeño académico de los estudiantes de nivel superior en Riversede", México. Revista de Investigación Educativa, 12.

JONASSEN, D.; HOWLAND, J.; MARRA, R. M. y CRISMOND, D. (2008): Meaningful Learning with Technology. Upper Saddle, River, N. J.: Pearson.

LEEDS, M., STULL, W. y WESTBROOK, J. (1998): "Do changes in classrooom techniques mater? Teaching strategies and their effects on teaching evaluations", Journal of Education for Business, 74, pp. 75-78.

LILLO, A. (2009): "El papel del capital humano en el sector turístico: algunas reflexiones y propuestas". Cuadernos de Turismo, $\mathrm{n}^{\circ} 24$, pp. 53-64.

LILLO, A., RAMÓN, A. y SEVILLA, M. (2007): "El capital humano como factor estratégico para la competitividad del sector turístico", Cuadernos de Turismo, no 19 , enero-junio, pp. 47-69, Universidad de Murcia.

MANZANO, M. (2007): Estilos de aprendizaje, Estrategias de lectura y su relación con el rendimiento académico del inglés como lengua extranjera. Tesis del programa de Doctorado "Aportaciones Educativas en Ciencias Sociales y Humanas". Universidad de Granada-Universidad de Ciego de Ávila.

MAQUILLÓN SÁNCHEZ, J. Y HERNÁNDEZ PINA, F. (2011):“Influencia de la motivación en el rendimiento académico de los estudiantes de formación profesional", REINFOP, vol. 14, $\mathrm{n}^{\circ} 1$, pp. 81-100.

MCROBBIE, C. y TOBIN, K. (1997): "A social constructivist perspective on learning environments”, International Journal of Science Education, vol. 19, pp. 193-208.

MEDINA, A. (2001):"Los métodos en la enseñanza universitaria”, en A. GARCÍA-VALCÁRCEL (coord.) Didáctica universitaria, pp. 155-198, Madrid, La Muralla.

MONROY, F. y HERNÁNDEZ-PINA, F. (2014): "Factores que influyen en los enfoques de aprendizaje universitario. Una revisión sistemática”, Educación XXI, vol. 17, n 2 , pp. 105-124.

OCAÑA, Y. (2011): "Variables académicas que influyen en el rendimiento académico de los estudiantes universitarios", Investigación Educativa, vol. 15, n 27, pp. 165-179.

PAL, R. y BUSING, M.E. (2008):"Busing Teaching operations management in an integrated format: Student perception and faculty experience international”, Journal Production Economics, pp. 594-610.

RINAUDO, M.C.; CHIECHER, A. y DONOLO, D. (2003): "Motivación y uso de estrategias en estudiantes universitarios. Su evaluación a partir del Motivated Strategies Learnig Questionnaire", Anales de Psicología, vol.19, n 1, pp. 107-119.

RODRÍGUEZ-MARTÍN, A. y ÁLVAREZ ARREGUI, E. (2013): “Metodologías y recursos en las titulaciones de Grado: Perspectiva de estudiantes y responsables institucionales", Revista Electrónica Interuniversitaria de Formación del Profesorado, vol. 16, $\mathrm{n}^{\mathrm{o}} 2$, pp. 105-120.

SIEGLE, D., RUBENSTEIN, L.D. y MITCHELL, M.S. (2014): “Honors Students' Perceptions of Their High School Experiences: The Influence of Teachers of Student Motivation",Gifted Child Quarterly, vol.58, n 1, pp. 35-50.

SIEGLE, D. y MCCOACH, D.B. (2005): Motivating gifted students. Wzco, Tx: PrufockPress. 
TEJEDOR, F., PONTES, C. RAMÍREZ, Y. y TEJADA, A. (2015): “Efectos de los métodos de enseñanza innovadores en el rendimiento académico de los estudiantes", $E K S$, vol. 16, no 2, pp. 109-121.

TEJEDOR, F.J. y GARCÍA-VALCÁRCEL, A. (2007): “Causas del bajo rendimiento del estudiante universitario (en opinión de los profesores y alumnos). Propuestas de mejora en el marco del EEES", Revista de Educación, 342, pp. 443-473.

TOMÁS-MIQUEL, J.V., EXPÓSITO-LANGA, M. y SEMPERE-CASTELLÓ, S. (2014): "Determinantes del rendimiento académico en los estudiantes de Grado. Un estudio en Administración y Dirección de Empresas", Revista de Investigación Educativa, vol. 32, n², pp. 379-392.

ULLAH, M.I., SAGHEER, A., SATTAR, T. y KHAN, S. (2013): "Factors Influencing Motivation to Learn in Bahauddin Zakariya University, Multan (Pakistan)", International Journal of Human Resource Studies, vol. 3, n 2, pp. 90-108.

URIEL, E. y ALDÁS, J. (2005): Análisis Multivariante Aplicado, Thomson Editores Spain, Madrid.

WILLCOX, M.R. (2011): "Factores de riesgo y protección para el rendimiento académico: Un estudio descriptivo en estudiantes de Psicología de una universidad privada", Revista Iberoamericana de Educación, $\mathrm{n}^{\circ}$ 55/1, pp.1-9.

WOLTERS, C.A. y PINTRICH, P.R. (1998): "Contextual differences in student motivation and self-regulated learning in mathematics, English, and social studies classrooms! Instructional Science, vol. 26, $\mathrm{n}^{\circ} 1-2$, pp. 27-47.

YU, D.D. (2011): "How Much Do Study Habits, Skills, and Attitudes Affect Student Performance in Introductory College Accounting Courses?", New Horizons in Education, vol. 59, no 3 , pp. 1-15. 